\title{
ACUTE AORTIC DISSECTION: A POSSIBLE HIGH RISK SCENARIO OF PERIPARTUM
}

Authors: Galveias I. ${ }^{1}$, Costa Rodrigues C. ${ }^{1}$, Damas M. ${ }^{2}$, Lança F. ${ }^{2}$, Encantado V. ${ }^{2}$

${ }^{1}$ Instituto Português de Oncologia, Dept of Anaesthesiology, Lisboa, ${ }^{2}$ Hospital de Santa Maria, Dept of Anaesthesiology, Lisboa Head of department- Dr. Lucindo Ormonde

\section{Background}

- Aortic dissection (AoD) during pregnancy is rare and life-threatening for both mother and fetus (I)

- According to the International Registry of Acute Aortic Dissections, the peripartum period of pregnancy confers a risk for AoD of $0,2 \%(2)$

- Aortic dissections often develop among individuals with connective-tissue disorders associated with abnormalities of the aortic wall, such as those present in familial thoracic aortic aneurysm/dissection, Marfan syndrome, Loeys-Dietz syndrome, vascular Ehlers-Danlos syndrome, and bicuspid aortic valve disease or Turner syndrome (3)

- Type A aortic dissection is the presence of dissection proximal to the left subclavian artery (Stanford classification)

- Aortic dissection is considered a surgical emergency and if untreated the mortality rate is extremely high

\section{Case Report}

42-year-old women, ASA II, submitted to a c-section at 34 weeks per non tranquilizing CTG, with no significant past medical history

\section{Pre and Intraoperatively}

Hemodynamic stability

Asymptomatic

$\checkmark$ General anesthesia

\section{4h after surgery}

$\checkmark$ Initiated an episode of dyspnea, refractory hypoxemia and bilateral crepitations. No chest pain

$\checkmark$ Patient was promptly intubated, ventilated and transferred to the intensive care unit

$\checkmark$ A thoracic CT scan confirmed a Type-A dissection involving ascending aorta

\section{Management}

\section{Emergent surgical intervention}

Repair of aortic dissection was carried and the ascending aorta was replaced by a tubular prosthesis

$\checkmark$ Postoperative course remained uneventefull and patient recovered fully

\section{Conclusions}

- It has been reported that pain is the most common symptom or complaint at the onset of Aod

- Although the patient's symptoms were not suggestive of aortic dissection the diagnosis was made in the least possible time

- Echocardiography and CT scan were determinant to make a differential diagnosis from acute pulmonary embolism

\section{Learning Points}

Pregnancy is an independent risk factor for aortic dissection. Although the clinical manifestations of acute aortic dissection are well described, the diagnosis is often overlooked in pregnancy. 